\title{
Implementation of a National Framework to Promote Health Data Sharing
}

\section{The German Medical Informatics Initiative}

\author{
Petra Knaup', Thomas M. Deserno², Hans-Ulrich Prokosch ${ }^{3}$, Ulrich Sax ${ }^{4}$ \\ 1 IMIA Representative of GMDS 2011-2017, Institute of Medical Biometry and Informatics, \\ University of Heidelberg, Heidelberg, Germany \\ 2 IMIA Representative of GMDS since 2018, Peter L. Reichertz Institute for Medical Informatics, \\ University of Braunschweig and Hannover Medical School, Braunschweig, Germany \\ ${ }^{3}$ GMDS Medical Informatics Board, Chair of Medical Informatics, University of Erlangen-Nürnberg, \\ Erlangen, Germany \\ 4 GMDS Medical Informatics Board, Department of Medical Informatics, University Medical Center \\ Göttingen, Georg-August-University Göttingen, Germany
}

\section{Introduction}

Sharing health data has been a major topic in medical informatics research in Germany in the last two decades. The latest developments show that the interdisciplinary collaboration between the fields of medical bioinformatics and systems medicine has remarkably progressed, and innovative solutions to foster health data sharing have been presented by various German research groups, e.g., requirements for data integration platforms $[1,2]$, the role of medical informatics for systems medicine $[3,4]$, the interconnection of system architectures for rare disease registries [5], or information technology (IT) supported patient recruitment [6].

However, the various types of heterogeneous health data produced by patient care and research turned out to be insufficiently integrated $[7,8]$. Often, research data only show a molecular snapshot of an individual disease. Intelligent correlation with clinical data is expected to offer new potential for patient care and biomedical research. Medical data semantic integration and joined analysis may not only lead to a better prediction of individualized decisions but also to a better understanding of the disease, and can be the base for new individualized prevention, diagnosis, and therapeutic measures.

Therefore, the German Ministry of Education and Research (BMBF) has launched the Medical Informatics Initiative (MI-I) $)^{1}$ to translate data sharing potential into effective practical use and to solve the prerequisites of data sharing like patient consent and semantic interoperability, starting with university medical centers but already being designed to be rolled out to all hospitals and to outpatient care organizations throughout Germany in the later stages of the program.

\section{The German Medical Informatics Initiative (MI-I)}

So far, BMBF has allocated 150 million Euros for the next four years to support this future-oriented initiative. The MI-I is expected to strengthen biomedical research and improve patient care. Innovative IT-solutions should facilitate the digital exchange and the intelligent use of data from patient care as well as clinical and biomedical research.

\section{German MI-I Consortia}

Based on an international review of comprehensive technical and methodological proposals, four inter-disciplinary consortia

\footnotetext{
Shared data, shared benefits, Medical Informatics: strengthening research, improving healthcare http://www. medizininformatik-initiative.de/en/start
}

have been selected for funding. Each of them has three to eight university medical centers as core partners with the goal of establishing data integration centers. The consortia and their participating university medical centers are:

- Data Integration for Future Medicine (DIFUTURE): Technical University Munich Medical Center, Ludwig-Maximilians-University Munich Medical Center, University Hospital Tübingen, and University of Augsburg Medical Center

- Heidelberg - Göttingen - Hannover Medical Informatics (HiGHmed): Heidelberg University Hospital, University Medical Center Göttingen, Hannover Medical School

- Medical Informatics in Research and Care in University Medicine (MIRACUM): University Hospital Erlangen, University Hospital Frankfurt, University Hospital Freiburg, University Hospital Gießen, University Medical Center Mainz, University Hospital Mannheim of Heidelberg University, University Hospital Magdeburg, University Hospital Marburg

- Smart Medical Information Technology for Healthcare (SMITH): University and University Hospital Leipzig, University Hospital Aachen, University Hospital Jena

Since it is an open initiative, all consortia should integrate new partners as from 2018 to enable the widespread use of the resulting 
solutions. In the future, researchers, physicians, and patients shall benefit from the initiative on a nationwide basis. The funding scheme is modular in several steps to enable flexible adaption to future developments. Rigorously scheduled audits will monitor and control the process.

\section{Medical Data Integration Centers (DIC) and Use Cases}

Every funded university medical center is expected to establish a local Data Integration Center (DIC), make this DIC accessible for shared data usage within the consortium and, on the long run, also for cross-consortial applications. DICs should enable data sharing and information exchange on a semantic level. The concept is based on decentralized, federated data hosting. Along the current ethic regulations and the EU General Data Protection Regulation (GDPR), use and access policies are effective. Special boards should decide by request whether for example an algorithm may calculate a result on local data, or aggregated data may be exposed, or actual data can be transferred via data transfer units.

DICs would demonstrate how data, information, and knowledge from patient care as well as clinical and biomedical research can be at least virtually integrated into a lifelong electronic health record (EHR) across different care providers, disregarding the types and vendors of EHR systems in use [9]. All consortia have defined medical use cases in order to prove the value of their DIC and to illustrate after a development period of three years that data sharing yields measurable improvements for patients and the society. MIRACUM, for example, has already demonstrated the benefit of guideline adherence in stroke patients [10]. Although these use cases were developed independently in the competitive phase, some remarkable overlap is observed. Several consortia work on clinical scenarios such as alerting in health care (infectiology), supporting molecular tumor boards (oncology), and supporting therapy decisions with clinico-molecular-based predictions. Other use cases are targeting improved research scenarios, for instance, by enhancing patient recruitment in clinical trials.
Furthermore, medical research shall benefit from better data and knowledge bases. Each consortium has defined key performance indicators to measure after three of the four years of funding, whether or not the objectives have been reached. Exemplary indicators are the number of patients who are treated in a cross-institutional therapy board or the number of patients who could be included within multi-center trials.

\section{National Coordination}

To make the MI-I a national and joint effort, a National Steering Committee (NSC) has been established to coordinate cooperation and foster interoperability between all funded consortia. The NSC elaborates joint agreements, initiates cross-consortial use cases, and ensures joint measures for interoperability. Each of the funded consortia is represented in the NSC by the consortia coordinator and a deputy: one professor of biomedical informatics and one dean or medical director of a university hospital. There are three central workings groups (WGs) with members from all funded consortia:

- WG "Patient Consent" works on a harmonized document (consented with the working group of German ethical committees and all German state data protection officers) to establish a broad patient consent that enables the privacy preserving use of clinical data for future research.

- WG "Data Sharing" works on the requirements for legally secure data access and data usage (a recommendation for governance structures and procedures for data use and access is one first result of this working group).

- WG "Interoperability" works on coordination issues to achieve interoperability between DICs. It has defined an interoperability roadmap and a first draft version of a core data set.

The funding scheme will be evaluated by the impact of the new governance, structures, and the global infrastructure on the health care system. Therefore, a roadmap was jointly developed describing all necessary tasks and milestones, e.g., comparable structured consent forms, standardized terminologies (e.g., SNOMED CT), clinical data models (e.g. openEHR, OMOP), use and access methods, and patient involvement.

\section{Teaching Medical Informatics for Sustainability}

The BMBF has already foreseen that specialized data scientists will be required to work in DICs once they are established to operate and analyze the increasing data generated by use cases and in clinical routine. In Germany, however, there are not enough existing medical informatics programs to meet all future requirements and the curricular content has to be updated with respect to new challenges. To strengthen medical informatics, all consortia have to work on these educational challenges. New curricula, digitalized learning modules, and novel educational concepts have to be developed. Harmonizing educational efforts and new contents will be a major issue for the NSC in close coordination with the respective working groups of the German Association for Medical Informatics, Biometry, and Epidemiology (GMDS). Digital online learning modules, for example, would be designed as modular and exchangeable components to be used in different study courses on different study levels. Additional substantial funding can be obtained when new professorships in biomedical informatics, data sciences, or related fields are established.

\section{Summary of the Road Map}

The goal of the MI-I is to achieve and maintain interoperability and the roadmap is based on four pillars: (1) standards, (2) coordination and iterative approaches, (3) intensive cooperation, and (4) risk management and monitoring.

The stepwise approach starts with milestones around the standardization of patient consent and data use agreement in different refinement steps. Therefore, trust centers have to be specified, a trans-consortia ID-management and modalities for record linkage have to be set up. In this context, privacy concepts, use and access rules, as 
well as use and access committees have to be specified and established. Furthermore, semantic interoperability spans across several milestones starting with a core data set with a basic module that builds on the existing German billing dataset, diagnosis and procedure codes, medication data, and laboratory data. Enhanced modules will be established in the funding years two to four focusing on data from, e.g., pathology, oncology, microbiology, and intensive care units. Cross-consortia feasibility queries and cohort identification would be supported based on elements from this core data set. In later phases, the role of the patient will be reviewed, as well as the need for training medical doctors and data scientists.

\section{Conclusion}

The German MI-I will advance the processing of heterogeneous data for research and patient care in Germany. New professorships have already been established that might leverage new biomedical research within innovative new areas of digital medicine (e.g., medical data science, big data technologies, digital patient involvement and patient engagement, sensor-based patient-generated data provision) and increase the number of biomedical informatics programs in Germany, thus producing more well-trained experts. The German MI-I has already shown to strengthen the collaboration between the university hospitals' IT departments and the (bio-)medical research institutes. Furthermore, interdisciplinary cooperation between the GMDS disciplines (medical informatics, bioinformatics, biostatistics, epidemiology, and health information management) has already been strengthened within the consortia especially during the nine months of the detailed concept development. Now it has to be shown that the concepts will indeed have a significant impact on both the quality of patient care and the efficacy of medical research.

\section{References}

1. Ganzinger M, Knaup P. Requirements for data integration platforms in biomedical research networks: a reference model. PeerJ 2015;3:e755.

2. Bauer CRKD, Ganslandt T, Baum B, Christoph J, Engel I, Löbe M, et al. Integrated Data Repository Toolkit (IDRT). A Suite of Programs to Facilitate Health Analytics on Heterogeneous Medical Data. Methods Inf Med 2016;55(2):125-35.

3. Bauer CR, Knecht C, Fretter C, Baum B, Jendrossek S, Rühlemann M, et al. Interdisciplinary approach towards a systems medicine toolbox using the example of inflammatory diseases. Brief Bioinform 2017;18(3):479-87.

4. Gietzelt M, Löpprich M, Karmen C, Knaup P, Ganzinger M. Models and Data Sources Used in Systems Medicine. A Systematic Literature Review. Methods Inf Med 2016;55(2).

5. Deserno TM, Haak D, Brandenburg V, Deserno V, Classen C, Specht P. Integrated image data and medical record management for rare disease registries. A general framework and its instantiation to the German Calciphylaxis Registry. J Digit Imaging 2014;27(6):702-13.

6. Trinczek B, Köpcke F, Leusch T, Majeed RW, Schreiweis B, Wenk J, et al. Design and multicentric implementation of a generic software architecture for patient recruitment systems re-using existing HIS tools and routine patient data. Appl Clin Inform 2014;5(1):264-83.

7. Dixon BE, Vreeman DJ, Grannis SJ. The long road to semantic interoperability in support of public health: Experiences from two states. J Biomed Inform 2014;49:3-8.

8. Berges I, Bermúdez J, Illarramendi A. Toward semantic interoperability of electronic health records. IEEE Trans Inf Technol Biomed 2012;16(3):424-31.

9. Maier C, Lang L, Storf H, Vormstein P, Bieber R, Bernarding J, et al. Towards Implementation of OMOP in a German University Hospital Consortium. Appl Clin Inform 2018; 9(1):54-61.

10. Haverkamp C, Ganslandt T, Horki P, Boeker M, Dörfler A, Schwab S et al. Regional Differences in Thrombectomy Rates: Secondary use of Billing Codes in the MIRACUM (Medical Informatics for Research and Care in University Medicine) Consortium. Clin Neuroradiol 2018. 